\title{
PROPIEDADES EMERGENTES, VALOR INTRÍNSECO Y ECOLOGÍA: ALGUNAS PERSPECTIVAS ÉTICAS SOBRE LOS HABITANTES DE LA ISLA NAVARINO
}

ESTEBAN CÉSPEDES

\section{RESUMEN}

Según una de las tesis centrales de la ética biocultural, en todo ecosistema o cultura, los hábitats, los habitantes y sus hábitos se determinan mutuamente. Un principio inspirado en esta idea es aquí caracterizado y denominado principio de codeterminación ecológica. Se muestra su relevancia para la ética ambiental, considerando cómo está vinculado con algunos de los estudios ecológicos enfocados en la región subantártica de Magallanes. Como se argumentará brevemente, algunos aspectos relacionados con el mencionado principio merecen ser aclarados desde una perspectiva ética y filosófica. Uno de los principales objetivos de este escrito es sugerir que los conceptos de fenómeno emergente y de valor ético intrínseco proveen una base teórica para la aclaración de tales aspectos.

PALABRAS CLAVE: ética ambiental, ética biocultural, valor ético, Filosofía Ambiental de Campo.

\section{EMERGENT PROPERTIES, INTRINSIC VALUE AND ECOLOGY: SOME ETHICAL PERSPECTIVES ON THE INHABITANTS OF NAVARINO ISLAND}

\begin{abstract}
According to one of the main theses of biocultural ethics, in every ecosystem or culture, habitats, inhabitants and habits are mutually determined. A principle inspired by this thesis is here characterized and called principle of ecological codetermination. Its relevance for environmental ethics is shown by considering how it is associated with ecological studies focused on the sub-Antarctic region of Magallanes. As briefly argued, some aspects related to the mentioned principle must be clarified from an ethical and philosophical perspective. One of the main goals of this work is to suggest that the concepts of an emergent phenomenon and of intrinsic value provide a theoretical basis for the clarification of such aspects.
\end{abstract}

KEY WORDS: environmental ethics, biocultural ethics, ethical value, Field Environmental Philosophy. 


\section{ÉTICA BIOCULTURAL Y EL PRINCIPIO DE CODETERMINACIÓN ECOLÓGICA}

Tradicionalmente, la ética se dedica al estudio de las acciones humanas, de sus consecuencias, de principios generales que puedan guiarlas y de la manera en que deben ser valoradas. Notablemente, en el marco general de la ética ambiental, no sólo deben ser consideradas las consecuencias que afectan a personas humanas cuando una acción es evaluada moralmente, sino también las consecuencias que afectan a individuos de otras especies y al entorno que habitan. Según la perspectiva de la ética biocultural (Rozzi, 2013), las acciones dependen de hábitos, los cuales se constituyen a partir de las interrelaciones entre habitantes y hábitats. El entorno no es sólo algo que debe ser considerado al evaluar una acción éticamente, sino que es también un factor condicionante de las acciones mismas. Además, los individuos no son simplemente realizadores de acciones que podemos analizar éticamente. Son también elementos constituyentes de su hábitat y del hábitat de otros. Es posible plantear esta codeterminación entre las partes de un ecosistema bajo el siguiente principio:

Principio de codeterminación ecológica. Los hábitos de un individuo dependen, en cierta medida, tanto de los individuos que están relacionados con su hábitat como del hábitat mismo. La vida de todo habitante depende tanto de sus hábitos como de otros habitantes. A la vez, todo hábitat está constituido tanto por sus habitantes como por los hábitos de sus habitantes.

Es preciso señalar que un hábito puede ser entendido como un tipo de conducta adquirida o como una disposición biológica innata. Ya que la codeterminación tripartita recién caracterizada ocurre tanto en el nivel ontológico como en el axiológico (Rozzi, 2013), podemos considerar dos subprincipios distintos. Pensemos primero en un principio ontológico de codeterminación, es decir, un principio según el cual esta relación es interpretada como una propiedad real de un sistema biológico o cultural.

Principio ontológico de codeterminación. En todo sistema biológico o cultural $S$ se cumplen las siguientes condiciones: a) Los hábitos de un habitante de $S$ dependen tanto de su hábitat formado en $S$ como de las acciones de otros habitantes de $S$.

b) La vida de todo habitante de $S$ depende de sus hábitos, del hábitat y de otros habitantes.

c) La constitución de todo hábitat en $S$ depende de los habitantes de $S$ y de sus hábitos.

Este principio permite estudiar ecosistemas analizando diferentes factores relevantes $y$ superando una mirada estática. Pensemos ahora en un principio axiológico de codeterminación, es decir, un principio dirigido al estudio de los valores. Según este principio, la codeterminación es una relación con la que es posible asociar valores éticos a los factores considerados.

Principio axiológico de codeterminación. Desde un punto de vista axiológico, para todo sistema ecológico o cultural $S$ se cumplen las siguientes condiciones:

a) El valor ético asignado a los hábitos de un habitante de $\mathrm{S}$ depende tanto de consideraciones sobre su hábitat como de consideraciones sobre la manera en la que estos hábitos afectan a otros habitantes de $S$.

b) El valor ético asignado a un habitante de $S$ depende de consideraciones sobre sus hábitos, sobre su hábitat y sobre su relación con otros habitantes.

c) El valor ético asignado a un hábitat dentro de $S$ depende de consideraciones sobre los habitantes de $S$ y sobre sus hábitos.

Así, ni los hábitos, ni los individuos, ni sus hábitats pueden ser evaluados éticamente de forma aislada. Considerando esto, este principio posee sin duda un carácter contextualista que podría dar sustento a la siguiente tesis: Si una acción es éticamente correcta o no depende del contexto en el que es realizada y evaluada.

En la próxima sección me enfocaré en el principio axiológico de codeterminación ecológica, pensando en su importancia con respecto a cuestiones vinculadas a la ética ambiental. Los estudios en los cuales estará enfocada la discusión arrojarán luz sobre esto. 


\section{APLICACIONES DE LA ÉTICA BIOCULTURAL}

En esta sección serán considerados algunos estudios en los que se aplica el marco teórico de la ética biocultural. Tal aplicación está guiada por la metodología de la denominada Filosofía Ambiental de Campo. Esta metodología está basada en la investigación interdisciplinaria, en la composición de metáforas, en actividades de campo orientadas éticamente y en propuestas de conservación (Rozzi et al. 2008). Se discutirá a continuación de qué manera el principio axiológico de codeterminación ecológica puede ser pensado como un fundamento de estos estudios.

\section{El visón en la isla Navarino}

El trabajo de Ramiro Crego y colaboradores (Crego et al. 2018) es un ejemplo claro del principio axiológico de codeterminación expuesto en la sección anterior. En él explican cómo la introducción del visón norteamericano (Neovison vison) afecta el entorno biológico y cultural de la isla Navarino, proponiendo una perspectiva según la cual la biodiversidad de un ecosistema merece en ciertos casos una mayor valoración ética que los individuos. Esta perspectiva, que puede ser llamada ecocéntrica, justificaría el control de una "especie invasora" como el visón con el fin de conservar la complejidad biológica de un hábitat. Como apoyo, se introduce la metáfora de "los ojos del árbol". Sobre la base de registros fotográficos realizados por cámaras instaladas en un árbol, es posible promover un especial elemento poético: Podemos percibir lo que el árbol percibe y cómo él experimenta la lenta homogeneización biocultural (sensu Rozzi, 2013).

Es necesario considerar algunos aspectos relevantes al comparar el valor de determinados individuos con el valor del ecosistema que habitan. El visón, como especie, tiene un impacto negativo para el ecosistema de la isla Navarino. Ahora, esto no implica que debamos asignar a los individuos de esta especie un valor ético inferior al que asignamos al ecosistema. Para entender esto, debemos considerar la noción de valor ético intrínseco. Un valor intrínseco es un valor asignado con independencia de otros valores. En el ámbito de la ética ambiental, ejemplos firmes de características a las cuales podemos asignar un valor intrínseco son la vida y la capacidad de poseer intereses (cf. Taylor, 1981; Singer, 1993).

Considerando esto y la situación concreta de la presencia del visón en la isla Navarino, podemos afirmar lo siguiente: es posible asignar al visón, como especie, un valor negativo. Sus hábitos dañan el hábitat y su biodiversidad. Así, controlar esta especie es una medida éticamente justificable. Sin embargo, los visones, como individuos, poseen un valor intrínseco positivo. Considerando este problema ético, la reflexión sobre los posibles métodos de control ecológico es sumamente relevante. Éstos deben ser elegidos cuidadosamente, desde una perspectiva ética mediante la que sea posible realizar las distinciones conceptuales apropiadas.

\section{El valor ético en la cultura yagán}

Jaime Ojeda y colaboradores (Ojeda et al. 2018) abordan la noción de valor intrínseco y su complementariedad con la noción de valor instrumental, proponiendo superar la dicotomía entre ambos valores, en el contexto del estudio sobre los moluscos de los canales del Cabo de Hornos y su importancia en la cosmovisión yagán. Estos autores afirman que las distintas especies de moluscos poseen para la cultura yagán tanto un valor instrumental como un valor intrínseco. Un valor instrumental es asignado a una entidad en la medida en la que permite alcanzar un determinado objetivo (cf. Elliot, 2005). Ya que todo objetivo posee, como tal, cierto valor, el valor instrumental de una entidad está determinado por el valor de otra y, por lo tanto, no es un valor intrínseco.

Para los yaganes, el valor instrumental de los moluscos no está asociado únicamente a su valor alimenticio, sino también a su uso para la fabricación de herramientas y la realización de rituales. Con respecto al valor intrínseco asignado por los yaganes a los moluscos, Jaime Ojeda y colaboradores mencionan un caso relatado por Martin Gusinde (1986), quien observó el respeto que ellos demostraban ante las lapas con el fin de evitar ciertas consecuencias negativas que ocurrirían si decidieran no hacerlo. Resulta importante mencionar lo siguiente: es aceptable 
pensar que, como afirman los autores, este respeto es signo de una valoración más profunda que la valoración relativa al valor alimenticio. Sin embargo, es difícil entender en qué medida esto puede implicar que los yaganes asignaran un valor intrínseco a estos moluscos, considerando la amenaza que, según Gusinde, ellos deseaban evadir. Es muy importante destacar que los yaganes valoraban la vida de estos organismos sobre la base de las consecuencias que su muerte podría implicar. Indudablemente valoraban una característica a la que deberíamos atribuir un valor intrínseco, es decir, la vida. Sin embargo, las razones de dicha valoración no parecen estar vinculadas a la atribución de valor intrínseco. Podemos juzgar las acciones de los yaganes ante las lapas como acciones éticamente correctas, pero no podemos asumir que tales acciones hayan estado basadas en una valoración noinstrumental de tales organismos.

\section{Los insectos del río Róbalo}

Tamara Contador y colaboradores (Contador et al. 2018) consideran el concepto de valor intrínseco al estudiar a los invertebrados que habitan el río Róbalo e investigar sobre su crucial importancia para el entorno. La metodología mediante la cual observan a los individuos se diferencia radicalmente de las metodologías clásicas: si es posible, las observaciones son realizadas in situ y, si no es posible, los individuos son llevados vivos al laboratorio. Después de ser estudiados, pueden ser regresados al lugar del cual fueron extraídos. Como se ha mencionado, la vida es una de las características a las que podemos asignar un valor intrínseco. La metodología ética con la que se realiza esta investigación ecológica está claramente basada en este aspecto del concepto de vida.

Adicionalmente, Contador y colaboradores describen una actividad correspondiente al taller educativo denominado Taller Omora del Medio Ambiente, en la que los participantes pueden observar a los invertebrados desde cerca en encuentros cara a cara. Según argumentan, estos encuentros promueven la reflexión sobre la relevancia de los invertebrados del río Róbalo, permitiendo una mejor comprensión de sus valores intrínsecos. Tal comprensión está relacionada directamente con el hecho de que estos animales pueden ser considerados como habitantes que poseen hábitos mediante los cuales constituyen su hábitat. Esto muestra la manera en la que el principio de codeterminación ecológica contribuiría al reconocimiento del valor intrínseco de un individuo.

Resulta necesario plantear la siguiente pregunta: ¿Es la comprensión del principio de codeterminación ecológica suficiente para evaluar apropiadamente una acción desde el punto de vista ético? En particular, pensando en la metodología ética propuesta en el artículo de Contador y colaboradores, parece perfectamente concebible que una persona que conozca los factores relevantes involucrados en el hábitat de una especie en particular actúe sin considerar el valor ético intrínseco de los miembros de dicha especie. De hecho, esto es precisamente lo que ocurre en investigaciones sobre invertebrados que se guían por la metodología clásica, en las cuales los individuos son considerados como simples muestras que pueden ser depositadas en alcohol y ser desmembradas.

\section{Plantas consideradas como habitantes y como hábitats}

Según la ética biocultural, una valoración correcta de los diferentes organismos no sólo exige considerarlos como habitantes dentro de un hábitat, sino también como un grupo de cohabitantes para los cuales la colaboración es fundamental. Ésta es una de las ideas centrales expuestas en el estudio de Méndez et al. (2018), el cual está enfocado en el crecimiento de algunas plantas y musgos de las zonas altas de la isla Navarino. Por sobre el límite arbóreo, un gran número de especies desarrollan una forma de cojín. Esta forma contribuye a la vida de otras especies al generar un microhábitat con una gran disponibilidad de nutrientes en medio de zonas desérticas. Sobre esta base, en lugar de considerar a esta zona de manera tradicional como un desierto andino, se propone verla como un conjunto de jardineras subantárticas altoandinas. Siguiendo esta metáfora, resulta interesante notar que las plantas que desarrollan una forma de cojín son 
habitantes y a la vez hábitats. La propuesta de Méndez, Cavieres y Rozzi ofrece así una manera de comprender el principio axiológico de codeterminación ecológica, lo cual tiene implicancias de gran relevancia relacionadas con la ética ambiental.

Cabe profundizar en algunas de las ideas señaladas en el estudio, planteando la siguiente pregunta: ¿Cómo es posible, desde un punto de vista teórico, que un individuo pueda ser caracterizado, a la vez, como habitante y como hábitat? La respuesta a esta pregunta debe estar basada en conceptos apropiados para pensar en tal posibilidad. Además, es preciso enfocar la respuesta de tal manera que las nociones de hábitat y habitante sean adecuadas para la asignación de valor ético, es decir, que sea posible atribuir valores éticos a un individuo, por un lado, como habitante $y$, por otro, como parte fundamental de un hábitat. Por supuesto, estas cuestiones son de suma relevancia para el desarrollo de una ética ambiental.

\section{Diversidad, singularidad y \\ la acción de nombrar}

Otra importante consecuencia de la aplicación de la ética biocultural es la idea de que las especies no son simples categorías taxonómicas bajo las cuales es posible clasificar determinados ejemplares. Sobre esta base, Lily Lewis y colaboradores (Lewis et al. 2018) presentan el desarrollo de una actividad que fomenta la interacción directa, desde una perspectiva cultural y personal, con las plantas briófitas (por ejemplo, los musgos) y líquenes. En ella se explora el acto de nombrar como un proceso que tendría más implicaciones vinculadas a la valoración ética de los seres vivos que los procedimientos tradicionales de clasificación o de reconocimiento taxonómico.

Es preciso destacar que el proceso de nombrar considerado por Lewis y colaboradores no implica solamente la capacidad de individuar a los habitantes de un determinado ecosistema, sino también de crear y descubrir diversidad. De esta manera, singularizar implica diversificar. Al pensar en esta idea, es importante que nos enfoquemos nuevamente en la relación entre la valoración ética de los individuos y la valoración ética de entidades más generales, como las especies y los ecosistemas.
Este tema será abordado a continuación, junto con los otros problemas planteados en esta sección.

\section{VALORACIÓN DE PROPIEDADES EMERGENTES}

En esta sección será caracterizada brevemente la manera en la que un valor ético puede ser asignado a aspectos que surgen a partir de las interacciones entre los elementos de un sistema. Para entender esta idea, necesitamos caracterizar la noción de fenómeno emergente (cf. El-Hani \& Pereira, 2000):

Fenómeno emergente. Un hecho o proceso es denominado fenómeno emergente cuando se considera cómo ha surgido a partir de un aumento en la complejidad de las interacciones entre los elementos de un determinado sistema.

Todo fenómeno emergente está asociado al desarrollo de un sistema, es decir, de un conjunto cuyos elementos interactúan de alguna forma. Algunos ejemplos de procesos considerados fenómenos emergentes son los derrumbes, la extinción de una especie y los procesos mentales. Los derrumbes pueden surgir de la interacción entre un gran número de granos de arena, piedras y rocas. La extinción de una especie puede surgir de la interacción entre las especies de un ecosistema. Los procesos mentales surgen a partir de las interacciones entre un gran número de neuronas. Es preciso mencionar que, como tales, los fenómenos emergentes no son reducibles a las partes constituyentes del sistema en el cual surgen. De esta forma, el derrumbe de un cerro es más que un conjunto de granos de arena, piedras y rocas interactuando. Al describirlo con precisión, el tipo de vegetación que crece en el cerro en cuestión podría ser un factor relevante. La extinción de una especie en un ecosistema es más que un resultado de la interacción entre las especies que lo componen. Muchas veces, los factores geográficos son cruciales. Los procesos mentales de un individuo son más que un conjunto de procesos neuronales. En general, la interacción con el entorno es igualmente fundamental para el surgimiento y configuración de procesos mentales.

También podemos hablar de los elementos 
constituyentes de un sistema desde una perspectiva ética. Los elementos éticos básicos de todo grupo o sistema son los valores éticos intrínsecos atribuibles a sus miembros. Enfocándonos en los sistemas biológicos, supondremos que las características de ser un organismo vivo y de poseer intereses son dos características fundamentales a las que podemos asignar un valor intrínseco. La principal razón de esta suposición es que ninguna de estas dos características es instrumentalizable. Pensemos, por ejemplo, en una planta de tomate. Podría ser muy valioso para un agricultor que la planta se mantenga con vida durante un período de tiempo determinado. Sin embargo, podemos asumir que no es la característica de estar viva lo que ve el agricultor de valioso en la planta, sino lo que la planta produce, es decir, las consecuencias de que ésta esté viva. La vida de la planta posee, como tal, sólo un valor para la planta misma. De igual manera, los intereses y los deseos poseen un valor intrínseco. No pueden ser instrumentalizados. Pensemos, por ejemplo, en un político que busca el interés de los electores. Es posible que el hecho de que un gran número de electores lo prefieran implique un gran valor para él. Sin embargo, son las consecuencias del interés de los ciudadanos los factores que lo beneficiarían y no los estados mentales de interés como tales.

Ahora bien, un valor intrínseco no es sólo un valor no-instrumental, sino también, como fue mencionado anteriormente, un valor básico, es decir, un valor que desde cierta perspectiva no es un simple compuesto de otros valores éticos. No osbtante, se debe considerar que las características a las que asignamos un valor intrínseco, según lo expuesto, son en cierto sentido propiedades emergentes. La capacidad de desear algo, por ejemplo, es una propiedad cognitiva que surge a partir de un complejo conjunto de interacciones entre diferentes elementos involucrados en distintos niveles, por ejemplo, las redes neuronales, las acciones del individuo que posee dicha capacidad $y$ los sucesos que ocurren en el entorno. Estos sucesos pueden involucrar individuos que también poseen valor intrínseco (pensemos, por ejemplo, en las interacciones personales) y entidades que no poseen un valor ético intrínseco (por ejemplo, las moléculas).

Existen propiedades emergentes que, si bien no poseen un valor intrínseco, surgen a partir de sucesos que involucran características a las cuales sí podemos asignar un valor intrínseco. Las características de un ecosistema son excelentes ejemplos de tales propiedades. Por ejemplo, no parece plausible asignar valor intrínseco al grado de diversidad de especies de un determinado sistema, aun cuando ésta es una característica que ha surgido a partir de interacciones entre individuos que poseen valor intrínseco. La principal razón de esto es que, en principio, la diversidad es instrumentalizable. Pensemos por ejemplo, en el valor que un agricultor puede asignar a la diversidad de un sistema de policultivo. De cualquier forma, es interesante que además de surgir a partir de propiedades a las cuales podemos asignar un valor intrínseco, la diversidad también permite que estas propiedades puedan seguir siendo instanciadas. En términos más simples, la diversidad de especies de un determinado sistema depende de los seres vivos que lo habitan y, al mismo tiempo, es una condición para el desarrollo de estos seres vivos. Éste es el caso de muchas características de los ecosistemas. (Consideremos, por ejemplo, la estructura o los ciclos periódicos de un ecosistema). Un aspecto crucial relacionado con este punto es que tenemos buenas razones para asignar un gran valor ético a estas características, a pesar de que no podamos asignarles un valor intrínseco. Esta clase de valor puede ser caracterizada de la siguiente forma:

Valor ecológico. El valor ecológico es el valor asignado a una característica emergente que ha surgido a partir de interacciones entre individuos que poseen valor intrínseco y que permite además la auto-organización y conservación de estos individuos.

Cabe destacar que la noción de propiedad emergente es especialmente relevante para esta caracterización y, por lo tanto, para el estudio de la ética ambiental en general. Según una noción alternativa, más restrictiva, el valor ecológico de una característica podría estar determinado por la manera en la que ésta sustenta la vida salvaje o nativa (cf. Cole, 2000; Cordell et al. 2005). Esta noción resultaría plausible mediante la suposición de que, considerando un ecosistema determinado, podemos asignar un mayor valor ético a los miembros de especies nativas que a los miembros de especies no nativas. En una primera instancia, 
parece difícil pensar en alguna justificación ética sólida para realizar tal suposición; como individuos, los miembros de una especie no-nativa poseen el mismo valor intrínseco que los miembros de una especie nativa.

Considerando estas ideas, enfoquémonos a continuación en algunos de los problemas señalados en la sección anterior. Uno de estos problemas surge pensando en la llegada del visón a la isla Navarino: ¿Podemos asignar un mayor valor ético a un ecosistema que a los individuos que lo habitan? Si bien la existencia de un ecosistema depende de la existencia de sus miembros, seres vivos individuales, no sería plausible afirmar, fuera del plano metafórico, que los ecosistemas son seres vivos o que pueden tener intereses determinables de la manera en la que los intereses individuales lo son. En este sentido, parece difícil asignar un valor ético intrínseco a los ecosistemas. Sin embargo, podríamos asignar un valor negativo a los hábitos de una especie, si éstos implicaran un riesgo de gran magnitud para la biodiversidad del ecosistema que habitan. Esta valoración sería justificable ya que la biodiversidad es una característica ecosistémica a la que podemos asignar un gran valor ecológico. Ahora bien, esto no significa que debamos asignar un mayor valor a la biodiversidad de un ecosistema que a los individuos que lo habitan. La biodiversidad de un ecosistema es valiosa precisamente porque sustenta la vida de un gran número de individuos. Éste es un punto que posee gran relevancia para la toma de decisiones vinculadas con el medio ambiente en general $y$, en particular, con el caso del visón.

Planteemos ahora uno de los problemas vinculados al principio de co-determinación ecológica. Recordemos que este principio está constituido por dos subprincipios: el principio ontológico de co-determinación y el principio axiológico de codeterminación. El problema es el siguiente. Una comprensión profunda de las bases ontológicas de un ecosistema no garantiza, por sí sola, una apropiada consideración ética del mismo. Afirmar lo contrario implicaría argumentar sobre la base de la denominada falacia naturalista (cf. Kerr, 2000). Ésta es una clase de argumento según el cual se pretende derivar un conjunto de proposiciones normativas simplemente a partir de un conjunto de proposiciones descriptivas. Una reacción ante este problema podría consistir en añadir un conjunto de premisas normativas al repertorio de premisas descriptivas, en particular, premisas generales sobre las nociones de valor intrínseco y valor ecológico que permitieran el tránsito desde el ámbito ontológico al ámbito valórico correspondiente a situaciones concretas. Sobre la base de estas premisas y con suficiente evidencia no sólo sería posible asignar valores intrínsecos correctamente, sino también resolver problemas concretos relacionados con los valores éticos de las características emergentes de un ecosistema. Sin duda, el principio de codeterminación ecológica es crucial con respecto a este punto.

Enfoquémonos ahora en la posibilidad de considerar a un individuo como habitante de un ecosistema y, a la vez, como parte fundamental de un hábitat que ofrece las condiciones apropiadas para la vida de otros individuos. Con respecto a la noción de propiedad emergente, cabe destacar que, en ciertos contextos, un individuo puede ser caracterizado como parte constituyente de un ecosistema, mientras que en otros contextos, el mismo individuo podría ser caracterizado como un sistema de propiedades emergentes que surgen sobre la base de procesos físicos, biológicos y químicos. Como se ha señalado, el mencionado estudio de Méndez et al. (2018) provee excelentes ejemplos de esto. Considerando lo expuesto, la valoración ética de un individuo o de características ecosistémicas también depende del contexto. Una ética ambiental determinada puede exigir que una valoración ética incluya información sobre todos los contextos posibles. No podemos valorar, por ejemplo, a un musgo que crece sobre el límite arbóreo de la isla Navarino sólo como una parte constituyente del ecosistema; debemos valorarlo además como una parte fundamental del hábitat en el que viven miembros de otras especies.

$\mathrm{Si}$ bien los fenómenos emergentes pueden ser caracterizados como sucesos que surgen a partir de procesos físicos básicos, también es necesario pensar en el procedimiento epistemológico mediante el cual éstos son estudiados generalmente. En la mayoría de los casos, los fenómenos emergentes son observados inicialmente de forma directa. Son sencillamente algo que aparece y que resulta relevante. En una segunda etapa, es posible construir modelos que expliquen y describan el 
surgimiento mismo de tales sucesos. De todas formas, al ser representaciones idealizadas, estos modelos no pueden cumplir la función de eliminar la experiencia directa inicial que involucra el tipo de fenómeno percibido. El trabajo de Lewis et al. (2018) muestra la importancia de estas experiencias directas y cómo están relacionadas con la actividad de nombrar. Estudiar una representación idealizada de un fenómeno emergente es una experiencia muy distinta a la de percibirlo directamente. De la misma manera, estudiar, por ejemplo, taxonómicamente distintas especies de líquenes implica una experiencia muy distinta a la de observar ejemplares directamente con el fin de otorgarles un nombre. En sentido estricto, al estudiar teóricamente una especie se estudia un modelo abstracto e idealizado. En contraste, al observar un ejemplar es posible percibir su individualidad, lo cual puede contribuir a una investigación acerca de cómo sus características relevantes podrían ser consideradas propiedades emergentes. Además, es posible investigar en qué medida un individuo es parte constituyente de fenómenos emergentes de un nivel de organización más alto. Estas ideas son cruciales para la ética ambiental, especialmente para comprender el rol de las nociones de valor intrínseco y de valor ecológico.

\section{CONCLUSIÓN}

El principio axiológico de co-determinación ecológica, caracterizado en esta nota, puede ser considerado una de las bases de la ética biocultural. Según este principio, al asignar un valor ético a un miembro o elemento de un ecosistema, es preciso considerar a los miembros y elementos del ecosistema en su conjunto. Por supuesto, los elementos de un ecosistema no son entidades estáticas, razón por la cual los hábitos y las acciones poseen una relevancia fundamental. Los problemas prácticos vinculados a la ecología y a la conservación exigen comparar los valores de diferentes partes de un ecosistema, lo cual implica de alguna forma la constante aplicación del mencionado principio. Orientado por estudios ecológicos sobre especies y hábitats de la isla Navarino, el presente trabajo se enfoca en tal clase de problemas. Éstos pueden ser expresados con preguntas como las siguientes: ¿Podemos asignar a las características generales de un ecosistema un mayor valor ético que a los individuos que lo habitan? ¿Cómo podemos asignar un valor ético a características biológicas que no poseen un valor intrínseco? ¿En qué medida puede contribuir el encuentro directo con un individuo a una valoración ética apropiada? Se ha intentado argumentar brevemente que es posible abordar estas cuestiones mediante el concepto de propiedad emergente. Sobre la base de este concepto, se ha señalado que hay buenas razones para asignar un gran valor ético a las características de un ecosistema que sustentan características asociadas a valores intrínsecos.

\section{AGRADECIMIENTOS}

Este trabajo es resultado de una estadía de investigación que realicé durante mayo de 2016 en la Estación de Campo Parque Omora, Puerto Williams, y que fue financiada por CONICYT en el marco del proyecto FONDECYT de postdoctorado N. 3160180. Agradezco profundamente a Omar Barroso, Tamara Contador, Nancyrose Houston, Jaime Ojeda, Javier Rendoll, Ricardo Rozzi, Camila Saldías y a Elke Schüttler por compartir conmigo discusiones y experiencias muy valiosas.

\section{BIBLIOGRAFÍA}

Cole, D. N. (2000). Paradox of the primevil: Ecological restoration in wilderness. Ecological Restoration 18(2), 77.

Contador, T., Rozzi, R., Kennedy, J., Massardo, F., Ojeda, O., Caballero, P., Medina, Y.,... \& Lazzarino, S. (2018) Sumergidos con lupa en los ríos del Cabo de Hornos: Valoración ética de los ecosistemas dulceacuícolas y sus habitantes. Este volumen.

Cordell, H .K., Murphy, D., Riitters, K. H., \& Harvard, J. E. (2005). The natural ecological value of wilderness. En Cordell, Bergstrom \& Bowker (2005). The Multiple Values of Wilderness (pp. 205-249). USA, PA: Venture Publishing.

Crego, R., Ward, N., Jiménez, J., Massardo, F. \& Rozzi, F. (2018). Los ojos del árbol: percibiendo, registrando, comprendiendo y contrarrestando las invasiones biológicas en tiempos de rápida homogeneizacion biocultural. Este volumen.

El-Hani, C. N. \& Pereira, A. M. (2000). Higher-level descriptions: why should we preserve them. In 
Andersen, Emmeche, Finnemann \& Christiansen (eds.). Downward Causation (pp. 1-29). Denmark: University of Aarhus Press.

Elliot, R. (2005). Instrumental value in nature as a basis for the intrinsic value of nature as a whole. Environmental Ethics, 27(1),43-56.

Gusinde, M. (1986). Los indios de Tierra del Fuego. Tomo II: Los Yámana. Buenos Aires: CAEA.

Kerr, A. J. (2000). The possibility of metaphysics: Environmental ethics and the naturalistic fallacy. Environmental Ethics, 22(1),85-99.

Lewis, L., Gottschalk, C., Saldías, C., Mackenzie, R., Goffinet, B. \& Rozzi, R. (2018). Cultivando un jardín de nombres en los bosques en miniatura del Cabo de Hornos: extensión de la conservación biocultural y la ética a seres vivos poco percibidos. Este volumen.

Méndez, M., Cavieres, L. \& Rozzi, R. (2018). Jardineras subantárticas: conocimiento y valoración de la flora altoandina. Este volumen.

Ojeda, J., Mansilla, A., Rosenfeld, S., Contador, T.,
Massardo, F., González- Calderon, J. \& Rozzi, R. (2018). Interacciones bioculturales del pueblo yagán con las macroalgas y moluscos: una aproximación desde la filosofía ambiental de campo. Este volumen.

Rozzi R., Arango X., Massardo F., Anderson C., Heidinger K., Moses K. (2008) Field environmental philosophy and biocultural conservation: the Omora Ethnobotanical Park educational program. Environmental Ethics, 30(3), 325-336.

Rozzi, R. (2013). Biocultural ethics: From biocultural homogenization toward biocultural conservation. En R. Rozzi, S. T. A. Pickett, C. Palmer, J. J. Armesto \& J. B. Callicott (Eds,). Linking Ecology and Ethics for a Changing World: Values, Philosophy, and Action (pp. 9-32). Netherlands: Springer.

Singer, P. (1993). Practical Ethics. New York: Cambridge University Press.

Taylor, P. (1981). The ethics of respect for nature. Environmental Ethics, 3(3),197-218. 
